\title{
Variations
}

Variations

Revue internationale de théorie critique

18 | 2013

"La poésie est une arme chargée de futur »

\section{Pour une écoute syndicale}

Contre la «souffrance au travail », la prise de parole sur le travail

Un collectif syndical Sud Education en Rhône-Alpes

\section{(2) OpenEdition}

Journals

Édition électronique

URL : http://journals.openedition.org/variations/647

DOI : $10.4000 /$ variations. 647

ISSN : 1968-3960

Éditeur

Les amis de Variations

Référence électronique

Un collectif syndical Sud Education en Rhône-Alpes, "Pour une écoute syndicale », Variations [En ligne], 18 | 2013, mis en ligne le 31 mai 2013, consulté le 01 mai 2019. URL : http://

journals.openedition.org/variations/647 ; DOI : 10.4000/variations.647

Ce document a été généré automatiquement le 1 mai 2019.

Les ami•e•s de Variations 


\title{
Pour une écoute syndicale
}

Contre la « souffrance au travail », la prise de parole sur le travail

\author{
Un collectif syndical Sud Education en Rhône-Alpes
}

\section{NOTE DE L'AUTEUR}

La scène se passe dans un établissement public d'enseignement supérieur qui a connu ces dernières années de nombreuses et profondes restructurations (fusion d'établissements, passage aux « Responsabilités et Compétences Élargies » prévues par la loi LRU...). L'expérience relatée est celle de la section Sud Education de l'établissement.

\section{CHRONIQUE D'UN APPRENTISSAgE SYNDICAL}

N'allez pas vers les collègues seulement avec des tracts déjà rédigés. Allez-y aussi avec une feuille blanche et de quoi noter. ${ }^{1}$

1 Souffrance au travail, risques psychosociaux... Au début, on s'empare de ces questions un peu comme à regret. On ne sait pas vraiment comment attraper ces sujets qui semblent individualiser et "psychologiser" les problèmes, nous engluer dans des conflits interpersonnels indémêlables, parfois un peu infantiles et jamais très politiques ( «c'est elle qui a commencé", «il ne peut pas me saquer», etc.). Surtout, on a le sentiment d'avoir déjà tant à faire sur le plan syndical : la lutte des collègues précaires; les risques de privatisation de certains services; les primes distribuées en toute opacité comme autant de bonus à la docilité ; les réorganisations menées au pas de charge et sans concertation; les entraves de tous ordres à l'activité syndicale jusqu'à, parfois, l'intimidation assez brutale de certain-es d'entre nous; le recul des formes collégiales de décision à tous les échelons du nouvel organigramme, remplacées par des comités de pilotage et autres cellules de prospective aux discours monomaniaques et strictement abstraits; les actions salutaires du collectif féministe contre la violence sexiste qui prospère impunément en milieu universitaire... 


\section{Une réalité qui s'impose}

2 Mais, d'une part, les dispositions légales sur l'hygiène et la sécurité prévoient désormais un volet obligatoire de prévention des risques psychosociaux (RPS), et le sujet est donc à l'ordre du jour des Comités Hygiène et Sécurité et des Conditions de Travail où nous siégeons (CHSCT). D'autre part, et surtout, la souffrance est bien là. On s'y heurte chaque jour au détour d'un couloir ou d'un appel téléphonique. Un silence puis un sanglot au bout du fil: "Je ne peux plus..." Dans le hall, une collègue livide, terrorisée par la perspective de son entretien d'évaluation, semble sur le point de perdre connaissance. Une autre est arrêtée plusieurs jours après une crise de nerfs. Un autre encore voit sa tension monter de 12 à 17 en quelques semaines suite à l'arrivée d'un nouveau chef qui lui demande de rendre compte de son travail par tranches de cinq minutes. Le médecin l'arrête immédiatement et lui interdit de revenir au travail tant que la situation n'est pas hors de danger pour lui. Au bout d'un moment, l'atmosphère est si lourde, la souffrance est si palpable, qu'on vient chaque matin au travail avec la crainte de voir se déclencher un «syndrome France Télécom» dans l'établissement. Et en effet, quelques mois plus tard, un collègue va faire une tentative de suicide.

Il n'y a donc pas le choix, il faut investir cette question de la souffrance au travail...

\section{La prise en charge institutionnelle de la souffrance au travail : une approche piégée}

4 La première tentative est de s'investir dans les instances, en particulier au CHSCT et dans son groupe de travail consacré aux risques psychosociaux. Mais quelles sont les pistes envisagées dans ces instances paritaires, dont la Direction est partie prenante ? Mettre en place des consultations de psychologues, ou même un «numéro vert » d'appel au secours, bâtir des « indicateurs »... Or, si la construction d'indicateurs est utile dans une démarche statistique, le nombre d'agents est trop faible à l'échelle d'un établissement pour que ces indicateurs puissent avoir du sens. Isabelle Forno cible ainsi les « diafoirus des RPS » :

L'obsession de la mesure semble trouver là sa caricature. [...] Comme s'il s'agissait [...] de passer un temps infini à la construction d'un thermomètre en en oubliant l'urgence du traitement et surtout, de penser que sans mesure il n'est nulle action efficace possible. ${ }^{2}$

En somme, la prévention des risques psychosociaux telle que nous la propose l'institution est un vaste piège, parce qu'elle repose sur l'idée faussement évidente qu'il s'agit de détecter les plus fragiles et de leur venir en aide. On ne peut manquer de faire le parallèle avec la tentation si répandue de faire de la victime le problème dans les crimes de viol. Ce n'est pas le travail, son organisation qui sont au coeur des préoccupations, mais les individus qu'on va surveiller, soigner et au besoin exfiltrer des situations pathogènes. On retrouve là la critique que fait Yves Clôt des approches officielles actuelles de la souffrance au travail, cette obsession à vouloir «soigner les personnes là où c'est le travail qu'il faut soigner " , approche qu'il qualifie de " tentation hygiéniste » :

[...][L]a prévention des risques psychosociaux [...], en évacuant [...] les confrontations collectives qui s'imposent sur la qualité du travail, [...] contribue à la fragmentation [des] collectifs en entamant les possibilités qu'ont les professionnels de se reconnaître dans ce qu'ils font. $»^{4}$ 
Dès lors, on comprend qu'une prise en charge syndicale efficace de ces problèmes ne pourra se faire qu'en dehors de ces instances. Nous nous retrouvons en effet bien plus dans l'analyse de Philippe Davezies, qui montre en quoi, le travail étant par nature à la fois collectif et subjectif, c'est bien plutôt l'absence de démocratie dans le travail qui fait courir un danger mortel aux individu-es car elle les mutile doublement, dans leurs besoins d'expression individuelle et de coopération :

À l'origine de la souffrance au travail, il y a toujours un désaccord sur la façon de
travailler. Si l'accent est mis sur la seule dimension interpersonnelle, c'est parce
que ce désaccord se présente dans un contexte relationnel déséquilibré. Dans le
scénario type, le supérieur met en avant une défaillance sur tel ou tel point. Dans ce
mouvement, il met l'accent sur un critère de performance qui lui importe, mais il
ne se met pas personnellement en jeu; le travailleur, au contraire, est interpellé
dans son activité. Pour que le conflit se développe sous la forme d'un débat
susceptible de produire une évolution positive, il faudrait que le salarié puisse
revenir sur son activité afin d'expliciter les enjeux qui ne sont pas perceptibles à
partir de la position du chef, mais sont néanmoins pertinents au regard de son
expérience professionnelle. Or, il ne dispose ni des temps, ni des espaces de
discussion nécessaires à cette élaboration. De ce fait, il est en position de se sentir
attaqué dans ce qu'il est. Il vit la critique sur le mode intrusif, persécutoire.

7 A cette prise de conscience s'ajoute le fait que la Direction de notre établissement est particulièrement friande des nouvelles modes ou lubies managériales dont le caractère violemment pathogène est pourtant largement démontré par la littérature scientifique, qu'il s'agisse de nouvelles méthodes d'encadrement à grand renfort de tableaux de bord et de réorganisations permanentes sans prise en compte de l'activité réelle ${ }^{6}$, de "l'évaluation individuelle des performances", imposture dangereuse ${ }^{7}$, de procédurisation à outrance du travail et de démarches qualité abstraites qui conduisent à des dénis de réalité et mettent en péril l'équilibre psychique des individu-es au travail ${ }^{8}$. Comment dès lors l'institution qui met volontairement en place des organisations du travail dangereuses pourrait-elle faire de la prévention efficace?

\section{Pour une écoute syndicale ${ }^{9}$}

Pour autant, cette prise de distance avec l'approche institutionnelle de la prévention de la souffrance au travail ne dit pas encore quelles alternatives construire et sous quelles formes lutter «sur le terrain». Pour notre section syndicale profondément attachée à l'auto-organisation des luttes et sensible aux dangers du syndicalisme par délégation, que peut bien signifier l'auto-organisation de collègues isolés et psychologiquement démolis? Comment des "pétages de plombs» individuels pourraient donner lieu à des luttes collectives? Et quel pourrait être l'horizon de ces luttes?

9 Si nous voulons échapper au piège de la "traque aux maillons faibles ", il faut réussir à montrer que c'est bien le travail en lui-même qui violente les individu-es, que le plus souvent, ce ne sont pas les plus « fragiles » mais au contraire les plus solidement investies professionnellement qui risquent d'être démoli-es le plus brutalement lorsque le travail se met à dysfonctionner.

Philippe Davezies souligne à quel point il est crucial alors de faire l'effort de sortir des discours politiques généraux (la casse des services publics, le manque de moyens...) car ils ne sont pas explicatifs de ce qui se passe, concrètement, dans la tête de quelqu'un qui craque au travail. Ils n'aident en rien les collègues qui souffrent et ils donnent le 
sentiment d'un discours idéologique plaqué qui tourne en boucle. Il ne s'agit pas d'abandonner ce niveau général de discours et d'analyse, mais de faire l'effort de le compléter par la compréhension des mécanismes du «pétage de plombs » au niveau de l'individu-e. Comment l'intensification du travail, le nouveau management public, les restrictions de moyens, vont-ils, concrètement, violenter tel et telle individu-e à un instant donné ? La démarche s'inverse alors : ce qu'on cherche à détecter, ce ne sont pas les individus les plus fragiles, mais les postes de travail les plus dangereux, exactement comme on le fait pour la prévention des autres risques, bactériologiques, chimiques, etc. D'un point de vue syndical, on commence à se reconnaître un peu mieux dans la démarche.

11 Et pour déceler la dangerosité de ces postes-fusibles, les mieux placés sont ceux et celles qui les occupent et leurs collègues immédiat-es. L'enjeu devient alors de créer des espaces de parole aussi fréquents et libres que possibles: utilisation des heures mensuelles d'information syndicale transformées systématiquement en assemblées générales, lutte pour faire appliquer le droit de réunion par bureau ou par service présent dans la loi de modernisation de la Fonction Publique...

Laissez les collègues parler du boulot. Et s'ils paranoïent sur le chef ou le collègue, ramenez-les toujours au boulot: non pas en quoi untel les déteste ou les persécute, mais en quoi concrètement il les empêche de travailler comme eux pensent qu'ils devraient travailler. ${ }^{10}$

On a bien sûr tenté d'indiquer à la Direction qu'ouvrir des espaces de parole libre au sein des collectifs de travail serait sans aucun doute une piste prometteuse. Celle-ci nous a rétorqué que la démocratie existait bien au sein de l'établissement puisqu'elle prenait soin d'y organiser, une à deux fois par an, des assemblées générales des personnels. Le dispositif consiste à rassembler tous les personnels dans le grand amphithéâtre de l'établissement. Les dirigeants sont sur l'estrade et expliquent, en commentant des diapositives et des diagrammes à l'écran derrière eux, le bienfait de leur action, sur les mois écoulés ou sur tel point précis, puis : «A vous la parole !». Un micro baladeur est à disposition. Curieusement, bien peu de choses ressortent de ces moments où il s'agit de prendre la parole devant des centaines de collègues et après ces exposés préparés et formalisés par la Direction, en comparaison avec la richesse des analyses sur le travail que nous entendons au contact direct des collègues. On retrouve là, mutatis mutandis, l'approche théorisée par Oskar Negt et Alexander Kluge :

On coupe littéralement l'herbe sous les pieds (d'un ouvrier) si on le prive de toute possibilité de construire ses phrases en lien avec des choses sensibles, une situation concrète ou un interlocuteur. Cela s'applique aux assemblées et réunions au sein de l'entreprise ou à des témoignages devant le tribunal, où il est forcé de parler d'une manière abstraite, alors qu'il maîtrise bien mieux la description cohérente des relations dont il a fait l'expérience. ${ }^{11}$

\section{De la libération de la parole à l'action collective}

Quelques exemples récents viennent témoigner des possibilités d'action qui s'ouvrent dès lors que des espaces de parole ont été créés. Ces exemples concernent des collègues ouvriers et ouvrières dont les fonctions ne relèvent pas de l'enseignement et de la recherche. Dans l'Etablissement, ces services "périphériques" sont les premiers à souffrir des restrictions budgétaires et des politiques d'externalisation. 
14 A l'été 2011, des collègues du service entretien ont contacté deux syndicalistes, dont l'une membre de notre section, pour leur faire part de difficultés rencontrées dans leur travail. Nous avons rencontré la plupart des agentes du service lors de deux assemblées générales. Lors de la première assemblée générale sont apparues des tensions interindividuelles: la première phase de libération de la parole s'est traduite par l'expression de souffrances personnelles, physiques et morales, ainsi que de conflits entre collègues. Dans un second temps, avec l'aide des militantes extérieures au service, les collègues du service entretien ont mis en évidence leurs intérêts communs et ont signé quasiment à l'unanimité une plate-forme de revendications. Les revendications portaient à la fois sur le matériel, l'organisation de la journée de travail et l'encadrement irrespectueux et inégalitaire de leur supérieure hiérarchique. Alors que la lutte s'organisait au service entretien, nous avons essayé de susciter un soutien intersyndical. Mais des résistances fortes nous ont été opposées par certains syndicalistes. De leur point de vue, les problèmes du service étaient davantage imputables à des comportements individuels violents ou déloyaux qu'à une organisation du travail pathogène. Comme la parole initiale des collègues du service entretien ne prenait pas la forme de la revendication classique, leur malaise n'était pas perçu comme un problème syndical. Or il s'agit bien d'action syndicale, car lorsque dans un service, un grand nombre de travailleurs/euses "pètent des câbles", ce n'est pas un problème de personnes mais d'organisation du travail.

15 Ces assemblées générales ont mené à une grève du service de dix jours à l'automne 2011 qui s'est soldée par une victoire sur tous les points de revendication. Cette victoire a été obtenue avec le soutien des autres services. En effet, au début de leur grève, une assemblée générale de l'ensemble des travailleurs/euses de l'Etablissement a fait ressortir les problèmes communs à tous les services - surcharge de travail, encadrement problématique, absence de réunions de service, manque de temps et de moyens pour effectuer un travail satisfaisant. Cette assemblée générale a débouché sur une grève générale d'une journée, libérant du temps pour faire des assemblées générales dans chaque service et établir une plate-forme de revendications communes à l'ensemble des services. La plate-forme a été discutée avec le Président et la nouvelle organisation du travail proposée par le service entretien a été retenue pour ce service. Pendant et après le mouvement de grève, les collègues ont témoigné d'impacts positifs de la lutte sur leur vie privée et leur santé, par exemple lors d'une interview par une radio locale, où elles notaient l'arrêt de la prise d'anxiolytiques, l'amélioration des relations avec leur entourage proche, conjoint, enfants... À l'issue du mouvement, un nombre important de collègues ont adhéré au syndicat, dont certaines sont devenues des militantes actives.

Si cet exemple prend appui sur une démarche collective, d'autres exemples de lutte syndicale illustrent la mobilisation autour de la défense administrative de dossiers individuels. Début 2012, un collègue fait une tentative de suicide en-dehors de son lieu de travail. Alertée par sa famille, qui affirme un lien direct avec sa situation professionnelle, notre section commence alors à reconstituer tous les faits survenus l'année précédant son geste, afin de mettre en évidence la responsabilité de l'organisation du travail. Nous avons réalisé des entretiens individuels avec différentes personnes du service et établi une chronologie des faits qui, accumulés, s'étaient avérés déstabilisants, alors qu'isolément ils pouvaient paraître mineurs. C'est le fait d'entrer dans le détail de ces événements qui a permis de mettre au jour les carences de la Direction de l'Etablissement face aux nombreux signaux d'alerte qui avaient précédé ce geste. Résultat majeur de ce 
long travail d'enquête, présenté comme une enquête CHSCT, même s'il a été réalisé par les militant-e-s du syndicat au-delà des seul-e-s élu-e-s de cette instance, notre collègue a obtenu que son accident soit qualifié en accident de service par la Commission de réforme. En outre, l'enquête a déclenché la reconnaissance par les collègues de ce service des problèmes structurels du service. Actuellement, nous luttons avec ces collègues contre la réorganisation sans concertation de leur service et contre l'externalisation de leur mission.

\section{Mandibule puissante}

On a alors la surprise de constater que tirer le fil de la souffrance au travail via l'écoute syndicale permet de dévider toute la pelote des questions du travail. On a l'impression tout à coup d'avoir à notre disposition une puissante mandibule permettant " d'attraper " sous un jour nouveau les sujets syndicaux plus traditionnels. À la mâchoire supérieure du discours politique général, s'articule désormais une mâchoire inférieure des mille et uns faits concrets de la vie au travail : l'absurdité des indicateurs et des directives abstraites, l'intensification du travail, le recul de la collégialité, les dérives budgétaires, prennent chair dans des situations réelles, datées, documentées. La prise de parole permet d'affirmer des points de vue jusque-là inaudibles sur le travail, son contenu et son organisation, de se renforcer et de prendre confiance face au discours managérial, et partant de contester des choix de gestion dans des domaines bien plus étendus que la seule dimension psychosociale. On en tire du grain à moudre y compris pour " réveiller » le combat dans les instances, qui auparavant s'étaient largement vidées de leur substance et dérivaient de plus en plus vers de simples chambres d'enregistrement.

\section{DIFfICULTÉs}

\section{Se protéger comme « écoutant-e »}

18 Il y a des difficultés liées à la violence des sujets dont on parle. Les collègues qui parlent de leur quotidien, de leurs conditions de travail décrivent souvent des situations de grande souffrance : isolement, harcèlement par la hiérarchie, conséquences sur leur vie personnelle, leur santé, leur moral, découragement. Pour les militant-es syndical-es à qui illes s'adressent, ces récits ont aussi un impact émotionnel. Entre le premier moment où les personnel-les parlent de leur travail et le moment où cette parole est réinvestie dans la lutte collective, le risque est de se retrouver seul-es et désemparé-es face à la souffrance des collègues. Il arrive qu'il n'y ait qu'un-e ou deux militant-es présent-es lors des entretiens avec les collègues. Ces militant-es se retrouvent seul-es dépositaires de ce récit. Afin d'éviter le découragement, l'isolement, et la pression liée au sentiment de responsabilité de ces militant-es, il est nécessaire de construire au sein de la section syndicale des espaces dans lesquels prendre en charge collectivement l'impact de ce travail syndical sur les militant-es. Il faut par exemple réfléchir à comment informer le reste de la section de la situation d'un-e collègue, sans trahir sa confiance placée en un groupe restreint de militant-es, et sans la présenter comme un "dossier syndical » désincarné. « Prendre en charge collectivement » l'impact sur les militant-es au sein de la section syndicale, c'est aussi réfléchir directement aux types d'actions qu'appellent ces 
récits (actions juridiques, assemblées générales, grève, tracts...) afin de contrer le sentiment d'impuissance de militant-es initialement non formé-es à l'écoute.

Il y a actuellement dans l'organisation du travail peu d'espaces pour que les travailleur-es s'expriment sur leur quotidien (ce qui à la fois est une cause de dysfonctionnements des collectifs de travail, et contribue à renforcer la souffrance au travail). En l'absence d'espaces pour la prise de parole, les collègues sollicitent toujours les mêmes militant-es identifié-es comme disponibles pour recevoir cette parole. La création de tels espaces repose donc entièrement sur la section syndicale. Nous organisons des assemblées générales (par service ou sur l'établissement dans son ensemble) visant explicitement à parler de l'organisation du travail, dans lesquelles nous n'imposons donc pas d'ordre du jour préalable, contrairement aux assemblées générales syndicales plus traditionnelles. Ces espaces de prise de parole collective permettent aux personnel-les de se rendre compte qu'illes font face aux mêmes difficultés: les récits de personnes de différents services se répondent et la production d'une analyse des dysfonctionnements se trouve facilitée, et produite collectivement pendant l'assemblée générale. En revanche, ces assemblées générales se font en temps limité ( $1 \mathrm{~h}$ par mois et par agent autorisée), les personnes n'y ont pas un accès égal à la parole, et elles sont aussi investies par des syndicalistes « professionnels » qui freinent l'expression en monopolisant la parole et en proposant immédiatement des «solutions » institutionnelles. Les Assemblées Générales ne constituent donc pas à elles seules un espace de parole suffisant.

\section{Créer des espaces-temps identifiés pour la parole : la permanence comme moment privilégié}

20 Pour pallier cela nous avons mis en place une permanence syndicale hebdomadaire. I s'agit de formaliser un espace et un moment de prise de parole, à la fois pour les collègues et pour les militant-es. Pour les collègues savoir qu'il existe un espace pour parler de leur travail. Pour les militant-es le choix de circonscrire le travail d'écoute à ces permanences, et de se le répartir entre elleux. En pratique, il n'est pas si facile de créer un espace qui remplisse ces deux fonctions.

21 Pour les collègues, le terme même de "permanence ", renvoie aussi à d'autres pratiques syndicales. Les syndicats d'accompagnement utilisent les permanences pour traiter individuellement des cas. Pour certain-e-s collègues (au moins dans un premier temps), il peut donc y avoir une attente d'une prise en charge totale de leur dossier au cas par cas. Il est donc nécessaire d'expliciter les limites de notre action syndicale et les modalités choisies par la section : nous ne sommes pas un syndicat d'accompagnement, et la lutte se fait avec les personnes concernées. En revanche, même si les collègues viennent parfois à la permanence avec une attente de prise en charge totale par le syndicat pour des problèmes habituellement peu investis par les syndicats de lutte, telles que les primes ou les promotions individuelles, c'est souvent en discutant de ces questions qu'apparaissent des problèmes d'organisation du travail profondément enracinés.

Le choix de cette action syndicale prend du temps car le temps du récit est incompressible. Il s'agit souvent pour les collègues d'une première prise de parole alors qu'illes sont parfois dans une situation difficile depuis longtemps. Cette première mise en mots peut se présenter de manière désordonnée, s'arrêter sur des problèmes en apparence triviaux, et sembler répétitive. Pour les militant-e-s syndicaux qui sont friands d'analyse macroscopique du travail, de textes synthétiques basés sur l'explicitation des 
rapports de force et des systèmes d'oppression, cette description très détaillée et ancrée dans le quotidien et le vécu des personnes est source de frustration. La tentation est grande d"interrompre les récits en calquant des grilles d'analyse toutes préparées. Il faut pourtant accepter de prendre ce temps de mise en récit des problèmes et de la souffrance des travailleur/euses. L'analyse des dysfonctionnements structurels n'est efficace que si elle émane des travailleur-es et de leur vécu. Pour les militant-es cela nécessite un changement de point de vue et de pratiques : ce n'est plus l'analyse a priori qui prime mais le récit du vécu des personnel-les, qui impose sa temporalité.

\section{Conséquences sur la section syndicale}

Le temps passé à discuter avec les collègues représente donc une part importante du travail militant, et entraîne une modification du fonctionnement de la section. Notamment, le temps de permanence se substitue parfois au temps de réunion. Tou-te-s les militant-e-s se rendent disponibles pour la permanence pour que ce ne soit pas toujours les mêmes personnes qui soient présentes. Comme il est difficile de trouver des moments où nous sommes tou-te-s disponibles, la permanence sert alors à traiter les affaires courantes, en lieu et place d'une réunion. Mais c'est un espace particulier, où se côtoient des militant-e-s du syndicat et des personnes venues parler de leur travail. Cela a permis à des personnes de venir militer plus facilement, car il y a une sorte de continuum entre travailleur/euses non militan-t-es et militant-e-s. Dans le cas de ces personnes, venir discuter et prendre la parole sur leur travail à la permanence les a amenées à s'impliquer plus largement dans le syndicat.

Pourtant, faire cohabiter réunion et permanence en un même temps et un même lieu n'est pas sans inconvénients: la permanence est un espace de parole libre pour les personnel-les tandis qu'en réunion la parole est modérée et régulée, pour être égale pour toutes les personnes. Mélanger ces deux types d'organisation de la parole pèse à la fois sur la portée de ces permanences, et sur l'organisation du syndicat. Il peut être impressionnant pour les personnel-les de parler de leur travail au milieu d'une réunion syndicale. Les réunions perdent en efficacité car elles sont moins formalisées : il est plus difficile de tenir un ordre du jour, et de distribuer la parole équitablement.

Par ailleurs, parce qu'une partie importante du temps militant est consacré à la discussion avec les collègues, certain-e-s militant-es peuvent avoir le sentiment de «ne faire que ça ", de délaisser les autres champs d'actions qu'illes aimeraient pourtant investir. Au niveau de la section, le risque est de délaisser certaines luttes, faute d'énergie militante. Le temps consacré à l'écoute n'est pas passé à faire d'autres activités syndicales plus traditionnelles, et parfois plus valorisées au sein du milieu militant (écriture, distribution de tracts...). Il est donc nécessaire, au niveau syndical, de légitimer et valoriser ces pratiques militantes, et dans le même temps, de se donner des objectifs en amont, et de définir un équilibre entre les différents types d'actions militantes.

En somme, ce n'est plus le syndicat qui choisit les luttes : là où la parole est prête à jaillir, le syndicat se fait outil, caisse de résonance, accumulateur, et enveloppe protectrice où le discours sur le travail peut se construire, s'élaborer le plus tranquillement possible. 


\section{Hostilité d'autres syndicalistes}

Nous faisons également face à l'hostilité parfois virulente des autres syndicats, ou de certains syndicalistes "professionnels", habitués inconsciemment à monopoliser la parole et à choisir leurs chevaux de bataille, à décider ce qui est digne d'intérêt ou pas, ce qui est juste ou pas. Notre stratégie de l'écoute vient les mettre en question, parfois très violemment, jusqu'à les fragiliser psychologiquement et/ou provoquer des réactions de rejet violentes.

ailleurs, l'efficacité de cette action ne se traduit pas forcément dans les urnes. Au contraire, on voit des syndicats particulièrement opposés à cette démarche remporter des victoires électorales, alors qu'ils défendent le "principe hiérarchique que nous bafouons » et soutiennent «les chef-fes». Ceci menace à terme la représentativité de la section syndicale. Ce décalage entre la pertinence travail militant, et son impact dans les élections peut s'expliquer par le fait que ce type d'action syndicale n'est pas toujours visible de l'ensemble des travailleur-es du site.

\section{EN gUISE de CONCLUSION : PRENDRE LA PAROLE... ET LA gARDER ?}

Mais le plus difficile reste de durer, de faire durer la parole...

Une manière de fixer la formulation de la souffrance et des problèmes passe par l'écriture. Notre section syndicale a lancé la publication d'un journal qui vise à rassembler des textes et des caricatures produits par les personnels. À la différence d'un journal syndical traditionnel, ce 4 pages a pour but de laisser de la place à la parole des travailleurs/euses en tant que telle, parfois sur le mode de l'humour, et de ne pas consister uniquement d'analyses syndicales. On a d'ailleurs constaté que ce journal a été un outil immédiatement réapproprié par des collègues qui participent peu d'ordinaire à la rédaction de tracts ou de communiqués. C'est aussi une façon de diffuser les récits des dysfonctionnements, de les visibiliser, et de permettre à des collègues de sortir de l'isolement, en créant un objet qui puisse servir de point de départ à une discussion. Le caractère inédit de ce mode de communication dans l'établissement a d'ailleurs suscité de violentes réactions de la Direction.

31 On a tenté de montrer que la prise de parole se fait parce qu'il y a une souffrance, une énergie sous pression que le dispositif d'écoute syndicale permet de faire jaillir et de mettre en mots et en luttes. Mais ensuite, après la bataille, lorsque la souffrance est apaisée, lorsque la joie et la fierté sont là, on voudrait pouvoir se « démobiliser », revenir à un quotidien paisible, jouir précisément des victoires remportées. Or aussitôt, reculs sur les acquis, nouveaux écrasements managériaux, poids des mauvaises habitudes autoritaires reprennent le dessus. Cette question de la durée, de la préservation des acquis, de l'impossibilité d'être en permanence mobilisé-es, est sans doute la plus problématique dans la lutte. Au-delà des difficultés que nous avons soulevées, il y a un véritable enjeu qui en vaut la chandelle à créer des espaces permanents de discussion autour du travail, afin que se perpétue la lutte. 


\section{COMMUNIQUÉ DE LA SECTION SUD DE L'ÉTABLISSEMENT EN DÉCEMBRE 2012}

\section{Pourquoi Sud se retire du Groupe de Travail Risques Psychosociaux}

Lors du Comité Hygiène Sécurité Conditions de Travail (CHSCT) d'octobre dernier, nous avons annoncé que Sud se retirait du groupe de travail sur les risques psychosociaux (GTRPS).

Ce groupe de travail est censé aider le CHSCT sur la question de la prévention des risques psychosociaux dans notre établissement. Mais après plus d'un an et demi de fonctionnement, ses résultats sont plus que maigres : ils sont inexistants. Ce GT-RPS a consacré la plupart de ses réunions :

- à sa propre charte de fonctionnement

- à tenter de bâtir des « indicateurs »

- à discuter de prévention secondaire et tertiaire, c'est-à-dire ce qu'il faut faire quand les problèmes sont déjà là ou quand un accident a déjà eu lieu (consultation de psychologue, dispositif d'alerte pour les personnes en souffrance, etc.), en évacuant systématiquement les discussions sur la prévention primaire : ce qu'il faut faire pour que les problèmes n'apparaissent pas et qu'il n'y ait pas d'accident. Un peu comme si, sur les risques bactériologiques ou chimiques, on discutait uniquement de quoi faire en cas d'accident, mais jamais de quoi faire pour éviter les contaminations ou les explosions!

Ce n'est pas faute d'avoir tenté de réorienter les travaux de ce groupe à maintes reprises...

En mai 2011, suite à une réunion qui nous paraissait partir dans la mauvaise direction, nous transmettons à la Direction le dossier « Stress au travail : la prévention fait fausse route » du magazine Santé et travail qui pointait déjà tous ces risques d'enlisement et expliquait :

Les entreprises en restent encore à l'élaboration d'un diagnostic ou à des mesures d'accompagnement individuel. Pourtant, tous les rapports sur le stress rédigés ces dernières années ont mis l'accent sur la profondeur de la crise du travail lui-même et sur la nécessité d'agir sur son organisation ${ }^{12}$. [...]

Un an plus tard, Sud intervient à nouveau, en questions diverses, au terme d'une réunion une nouvelle fois consacrée à la charte de fonctionnement du GT-RPS et aux indicateurs, pour réaffirmer la nécessité de travailler enfin sur la prévention primaire : quelles sont les méthodes d'encadrement et les formes d'organisation du travail connues pour être pathogènes et dangereuses ? Comment savoir si certaines ont cours actuellement dans notre établissement? Comment agir pour que les encadrant-es n'aillent pas vers ces formes dangereuses de management ou pour qu'ils les abandonnent au plus vite? 
Autant de questions élémentaires auxquelles le GT-RPS, en un an et demi de fonctionnement, n'a pas consacré une seule minute. [...]

Cette activité bureaucratique et vide se fera donc désormais sans Sud. [...]

\section{NOTES}

1. Bernard Bouché, syndicaliste Solidaires, formateur « Prise en charge syndicale de la souffrance au travail. »

2. Isabelle Forno, Travail, peurs et résistances. Critique de la victimisation du salarié, Editions Syllepse, Paris 2012, p. 69

3. Le travail à coeur, pour en finir avec les risques psychosociaux, p. 113

4. op. cit., p. 128.

5. Philippe Davezies, «Enjeux, difficultés et modalités de l'expression sur le travail : point de vue de la clinique médicale du travail», Pistes n¹4-2 2012 http://pistes.revues.org/2566

6. "L'organisateur a cédé la place au manager. L'organisateur prétendait connaître le travail ; le manager n'a plus cette prétention. Son bagage est constitué de savoir décontextualisés, sans lien avec les contenus techniques des activités qu'il doit encadrer: outils de gestion et fragments de sciences humaines constituant une sorte de technologie politique. [Mais] le désengagement des hiérarchies vis-à-vis des modalités d'exécution du travail ne s'est pas traduit par le déploiement d'un laisser faire généralisé. Le contrôle ne s'est pas relâché. Bien au contraire, il s'est plutôt accentué [...] Il est assumé par des individus plus éloignés du métier qu'autrefois et il repose sur des indicateurs de plus en plus abstraits. » Philippe Davezies (Université Lyon I), "Protéger et construire la santé au travail», intervention aux Journées Nationales de la Fédération d'Aide à la Santé Mentale Croix-Marine, 22 septembre 2003, Lyon.

7. «Les [...] évaluations individualisées des performances, sont [...] critiquables parce qu'elles sont arbitraires. [...][E]n effet, [...] il est facile de montrer que l'essentiel du travail n'est pas évaluable objectivement et quantitativement. Il s'ensuit forcément un sentiment confus d'injustice qui a aussi sa part dans l'apparition des décompensations, notamment à forme de syndromes dépressifs et de syndromes de persécution. » (C. Dejours, « Aliénation et clinique du travail », in Actuel Marx 2006/1 n³9)

8. "Les normes de la qualité totale [...] contraignent les travailleurs à dissimuler, plus encore que naguère, les infractions qu'ils doivent faire par rapport aux prescriptions, pour atteindre les objectifs (gestion de l'écart entre la conception de l'organisation du travail et la réalité du procès de travail). Tout le monde est ainsi progressivement appelé à participer à une description déformée et enjolivée de la réalité, qui occulte les infractions mais aussi le réel. (C. Dejours, "Aliénation et clinique du travail ", in Actuel Marx 2006/1 n³9)

9. Clin d'œil à Alexander Neumann, "Pour une écoute sociologique », Variations [En ligne], 8|2006, mis en ligne le 27 décembre 2012, consulté le 03 janvier 2013. URL: http:// variations.revues.org/501

10. Bernard Bouché, syndicaliste Solidaires, formateur «Prise en charge syndicale de la souffrance au travail. »

11. Oskar Negt, Alexander Kluge, Öffentlichkeit und Erfahrung, Suhrkamp, 1972, p. 87, traduit et cité par Alexander Neumann, "Pour une écoute sociologique, Répression, inhibition, prise de parole ", Variations $\mathrm{n}^{\circ} 8,2006$.

12. Santé et travail, avril 2011, $\mathrm{n}^{\circ} 074 \mathrm{http} / /$ www.sante-et-travail.fr/la-prevention-fait-fausseroute--in_fr_art_1084_53830.html 